15

\title{
Установка для измерения времени жизни нейтрона с большой гравитационной ловушкой при низкой температуре
}

\author{
(C) А.П. Серебров, ${ }^{1}$ Е.А. Коломенский, ${ }^{1}$ А.К. Фомин, ${ }^{1}$ А.О. Коптюхов, ${ }^{1}$ И.А. Краснощекова, ${ }^{1}$ \\ А.В. Васильев, ${ }^{1}$ Д.М. Прудников, ${ }^{1}$ И.В. Шока, ${ }^{1}$ А.В. Чечкин, ${ }^{1}$ М.Е. Чайковский, ${ }^{1}$ B.Е. Варламов, ${ }^{1}$ \\ C.Н. Иванов, ${ }^{1}$ A.H. Пирожков, ${ }^{1}$ P. Geltenbort, ${ }^{2}$ O. Zimmer, ${ }^{2}$ T. Jenke, ${ }^{2}$ M.G.D. van der Grinten, ${ }^{3}$ M.A.H. Tucker ${ }^{3}$
}

${ }^{1}$ НИЦ „Курчатовский Институт“ - ПИЯФ им. Б.П. Константинова, 188300 Гатчина, Ленинградская обл., Россия

2 Institut Max von Laue Paul Langevin, 71 avenue des Martyrs,

F-38042 Grenoble Cedex 9, France

${ }^{3}$ Science and Technology Facilities Council, Rutherford Appleton Laboratory, Harwell Campus, Didcot, Oxon, OX11 0QX, UK

e-mail: ivanov_sn@pnpi.nrcki.ru

(Поступило в Редакцию 20 марта 2018 г.)

Наиболее точное в настоящее время измерение времени жизни нейтрона проведено в ПИЯФ с удержанием ультрахолодных нейтронов в гравитационной ловушке. Представлена модернизация установки с большой гравитационной ловушкой с охлаждением до температуры $10-15 \mathrm{~K}$. Представлены результаты измерений температурной зависимости потерь ультрахолодных нейтронов при столкновении со стенками, покрытыми перфторированной пастой Fomblin UT 18 в диапазоне температур 300-77 К. Приведены оценки возможных теплопритоков к ловушке и показана возможность достижения указанного уровня температур в описанной конструкции.

DOI: 10.21883/JTF.2019.02.47089.118-18

\section{Введение}

Измерение времени жизни свободного нейтрона остается важной экспериментальной проблемой. Время жизни нейтрона напрямую используется в расчетах распространенности легких ядер при первичном ядерном синтезе в космологических моделях $[1,2]$. В комбинации с измерениями корреляционных коэффициентов в бета-распаде нейтрона время жизни нейтрона используется для определения векторной $G_{V}$ и аксиально-векторной $G_{A}$ констант слабого взаимодействия [3-5]. Эти константы, в свою очередь, необходимы для расчетов потоков солнечных и реакторных нейтрино [6,7], а также для проверки унитарности матрицы Кабиббо-Кобайяши-Маскавы в Стандартной Модели и поиска Новой Физики вне Стандартной Модели [8].

Для измерения времени жизни свободного нейтрона используются в основном два метода. В одном методе на пучке холодных нейтронов (скорость $v \approx 1000 \mathrm{~m} / \mathrm{s}$, энергия $E \approx 5 \mathrm{meV}$ ) регистрируются продукты распада нейтронов в области пролета и тем самым определяется число распавшихся нейтронов [9-12]. В другом методе ультрахолодные нейтроны (УХН) (скорость $v<7 \mathrm{~m} / \mathrm{s}$, $E<250 \mathrm{neV}$ ) удерживаются длительное время в специальной ловушке и регистрируется число нейтронов, оставшихся в ловушке после определенного интервала времени.

Наилучшая точность определения времени жизни нейтрона получена в ПИЯФ в эксперименте с гравитационной ловушкой $[13,14]$.
Для создания сосудов хранения нейтронов используется или отражение нейтронов от вещества с положительной длиной когерентного рассеяния [15-18], или отражение нейтронов при взаимодействии магнитного момента нейтрона с градиентом магнитного поля $[19,20]$, или комбинация этих методов с использованием взаимодействия нейтронов с гравитационным полем Земли [13,20-23]. В последнем случае кинетическая энергия нейтронов ограничена величиной потенциальной энергии в гравитационном поле, соответствующей высоте ловушки. Длительность удержания в принципе ограничивается распадом нейтрона. В идеализированном представлении процесса отражения нейтронов поверхность вещества представляется ступенчатым барьером с высотой, соответствующей величине оптического потенциала. Коэффициент отражения от поверхности зависит от компоненты кинетической энергии, определяемой составляющей скорости нейтрона, направленной перпендикулярно поверхности ловушки, и потерями в материале стенок ловушки, которые определяются мнимой частью нейтронно-оптического потенциала.

В эксперименте регистрируется число нейтронов $N_{1}$ и $N_{2}$, оставшихся в ловушке после различных времен удержания $t_{1}$ и $t_{2}$, и вычисляется постоянная времени удержания $\tau_{s t}=\left(t_{2}-t_{1}\right) / \ln \left(N_{1} / N_{2}\right)$ в предположении экспоненциальной зависимости числа нейтронов в ловушке от времени. Полную вероятность потерь УХН $\tau_{s t}^{-1}$ можно представить в виде суммы двух частей: вероятности бета-распада $\tau_{n}^{-1}$, не зависящей от столкновений нейтронов со стенками ловушки, и вероятности потерь в стенках ловушки $\tau_{\text {loss }}^{-1}$, зависящей от частоты 
столкновений нейтронов со стенками

$$
\tau_{s t}^{-1}=\tau_{n}^{-1}+\tau_{\text {loss }}^{-1} .
$$

Потери нейтронов в ловушке в процессе удержания, не связанные с бета-распадом, происходят за счет поглощения в материале стенок и из-за неупругих столкновений нейтронов со стенками ловушки, при которых происходит увеличение энергии нейтронов до величины выше нейтронно-оптического потенциала и соответственно выход нейтронов за пределы ловушки. Вероятность потерь нейтронов при столкновении со стенками определяется вероятностью потери при одном столкновении $\mu(E, T)$, зависящей от энергии нейтронов и температуры стенок ловушки, и частотой столкновений $v(E)$, зависящей от энергии нейтронов и геометрических размеров ловушки. В свою очередь, вероятность потерь можно представить в виде произведения фактора потерь $\eta(T)$, зависящего от свойств поверхности ловушки, но не зависящего от энергии нейтронов, на эффективную частоту столкновений нейтрона со стенками ловушки $\gamma(E)$, не зависящую от свойств поверхности, но зависящую от энергии нейтрона и геометрических размеров ловушки

$$
\tau_{\text {loss }}^{-1}=\mu(E, T) \cdot v(E)=\eta(T) \gamma(E) .
$$

Время жизни нейтрона, определяемое бета-распадом $\tau_{n}$, получается экстраполяцией измеренной постоянной времени хранения $\tau_{s t}$ к нулевому значению эффективной частоты столкновений. Различные значения эффективной частоты столкновений получаются или при использовании ловушек с различными геометрическими размерами (геометрическая экстраполяция), или при различной энергии нейтронов (энергетическая экстраполяция). Чем меньше фактор потерь $\eta(T)$, тем меньше вероятность потерь нейтронов $\mu(E, T)$ и тем ближе измеренная постоянная времени хранения к постоянной времени бета-распада и соответственно меньше диапазон возможных систематических ошибок в эксперименте.

Измерения времени жизни нейтрона с большой гравитационной ловушкой проводились на пучке УХН на высокопоточном реакторе Института Лауэ-Ланжевена в Гренобле (Франция) [14].

\section{Экспериментальная установка}

Установка с большой гравитационной ловушкой и методика измерений подробно описаны в работах $[23,24]$.

В нашем эксперименте время жизни нейтрона измеряется методом удержания нейтронов в ловушке с гравитационным затвором. Ловушка представляет собой полуцилиндр из меди с горизонтально расположенной осью. Когда ловушка находится в нижнем положении, нейтроны, находящиеся внутри ловушки, не могут из нее выйти, если их кинетическая энергия внизу ловушки недостаточна для подъема в гравитационном поле на

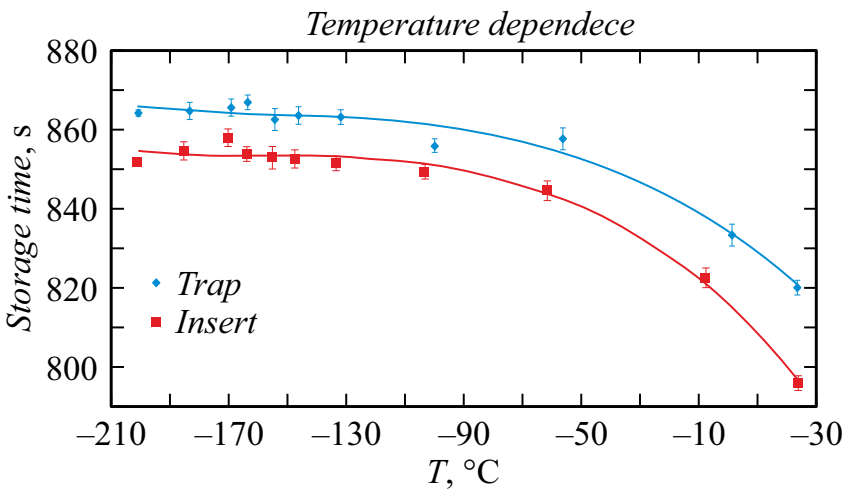

Рис. 1. Температурная зависимость постоянной времени хранения УХН в медной ловушке с перфторированным покрытиeм.

высоту больше или равную радиусу полуцилиндра. Такие нейтроны удерживаются внутри ловушки и могут выпускаться из нее при повороте ловушки. В нашей установке радиус ловушки равен $70 \mathrm{~cm}$ и в ней могут удерживаться нейтроны, энергия которых внизу ловушки не превышает $69 \mathrm{neV}$. Энергия выпущенных нейтронов определяется углом поворота ловушки. Внутренняя поверхность ловушки покрыта перфторированной пастой типа „Fomblin grease UT18“, которая имеет очень низкий фактор потерь при отражении УХН и хорошо наносится на металлическую поверхность. Граничная энергия отражения нейтронов для покрытия составляет $106 \mathrm{neV}$. Изменение эффективной частоты столкновений нейтронов с поверхностью осуществляется путем введения в ловушку дополнительной поверхности (вставки) в виде медного полуцилиндра с меньшими радиусом и длиной, чем у ловушки. Внутренняя и внешняя поверхности вставки покрыты тем же веществом. Экспериментальная установка и методика измерений подробно описана в работе [24]. Установка имеет два вакуумноизолированных объема. Корпус внутреннего объема, ловушка и вставка охлаждались до температуры $T=77 \mathrm{~K}$. Для этого корпус внутреннего объема имеет четыре емкости, в которые заливался жидкий азот. Две емкости использовались для охлаждения корпуса внутреннего объема, а две другие были соединены гибкими шлангами с трубками, имеющими тепловой контакт с ловушкой и вставкой для их охлаждения. Гибкие шланги позволяли поворачивать ловушку и вставку без нарушения вакуума. Охлаждение ловушки и вставки позволяет уменьшить потери при хранении нейтронов, так как сечение неупругого рассеяния нейтронов при столкновении с поверхностью, покрытой перфторированной пастой, уменьшается при понижении температуры. Измеренная зависимость постоянной времени хранения УХН в ловушке показана на рис. 1.

Зависимость фактора потерь УХН от температуры более явно видна на рис. 2, где из экспериментально измеренной постоянной времени хранения вычтены по- 


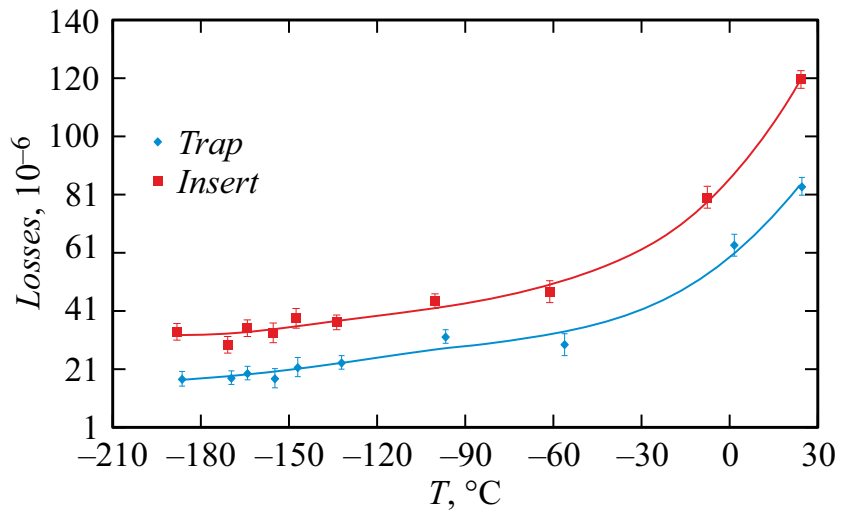

Рис. 2. Температурная зависимость коэффициента потерь УХН при столкновении со стенками ловушки со вставкой и без вставки.

тери, обусловленные бета-распадом нейтронов

$$
\tau_{\text {loss }}^{-1}=\tau_{s t}^{-1}-\tau_{n}^{-1} \text {. }
$$

Различие потерь для ловушки без вставки и со вставкой определяется различием в эффективной частоте соударений. Из графика видно, что фактор потерь при температуре $T=77 \mathrm{~K}$ продолжает уменьшаться с понижением температуры. Этот факт позволяет надеяться, что дальнейшее понижение температуры ловушки и вставки приведет к дальнейшему уменьшению фактора потерь из-за неупругого рассеяния и в результате постоянная времени хранения УХН будет ближе к постоянной времени бета-распада и позволит улучшить точность определения этой фундаментальной величины.

\section{Модернизация системы охлаждения}

Для проведения эксперимента при более низкой температуре предполагается модернизировать существующую экспериментальную установку с большой гравитационной ловушкой. Схема установки представлена на рис. 3.

Охлаждение ловушки и вставки предполагается проводить с помощью жидкого гелия. Для этого сделаны две емкости из нержавеющей стали объемом 1401 каждый, которые будут заполняться жидким гелием из транспортных дьюаров. Испаряющийся из емкостей газ, проходя по трубкам, присоединенным к ловушке и вставке в их верхней части, будет охлаждать их. В модернизированном варианте ловушка будет иметь тот же радиус $70 \mathrm{~cm}$, но меньшую длину $-170 \mathrm{~cm}$. Вставка, имеющая вид цилиндрической обечайки с радиусом $60 \mathrm{~cm}$ и длиной $150 \mathrm{~cm}$, может опускаться в ловушку. Ловушка и вставка имеют общую ось вращения типа труба-в-трубе и могут поворачиваться независимо друг от друга. Приводы вращения вставки и ловушки расположены на противоположных сторонах снаружи внешнего вакуумного кожуха. Ловушка и вставка снабжены свинцовыми противовесами для балансировки и уравновешивания сосудов хранения. Противовес ловушки закреплен непосредственно на ловушке внутри установки, а противовес вставки закреплен на валу снаружи внешнего вакуумного кожуха. Испаряющийся из емкостей гелий после прохождения по трубкам, закрепленным на ловушке и вставке, выводится наружу по гибким металлическим рукавам и уходит в линию для сбора газообразного гелия. Поток газа, проходящего по контурам охлаждения ловушки и вставки, регистрируется с помощью расходомеров отдельно для ловушки и вставки.

Приток тепла к ловушке и вставке происходит как за счет излучения от кожуха, находящегося при температуре $77 \mathrm{~K}$, так и за счет теплопроводности по валам, на которых поворачиваются ловушка и вставка. Теплообмен за счет остаточного газа во внутреннем объеме пренебрежимо мал, так как в установке поддерживается вакуум на уровне ниже $10^{-6}$ mbar. Точный расчет теплопритока к емкостям с жидким гелием, к ловушке и к вставке затруднителен по причине сложной геометрии поверхностей и приблизительного знания коэффициентов теплового излучения и лучепоглощения для холодных поверхностей, изготовленных их нержавеющей стали и меди, которые, кроме того, покрыты перфторированной пастой. Приблизительная оценка дает величину теплопритока к емкостям с жидким гелием за счет излучения примерно $1 \mathrm{~W}$. Дополнительный приток тепла за счет опор, конструкций крепления и подсоединительных гибких рукавов, предназначенных для заливки гелия и для отвода испаряющегося газа, не превышает

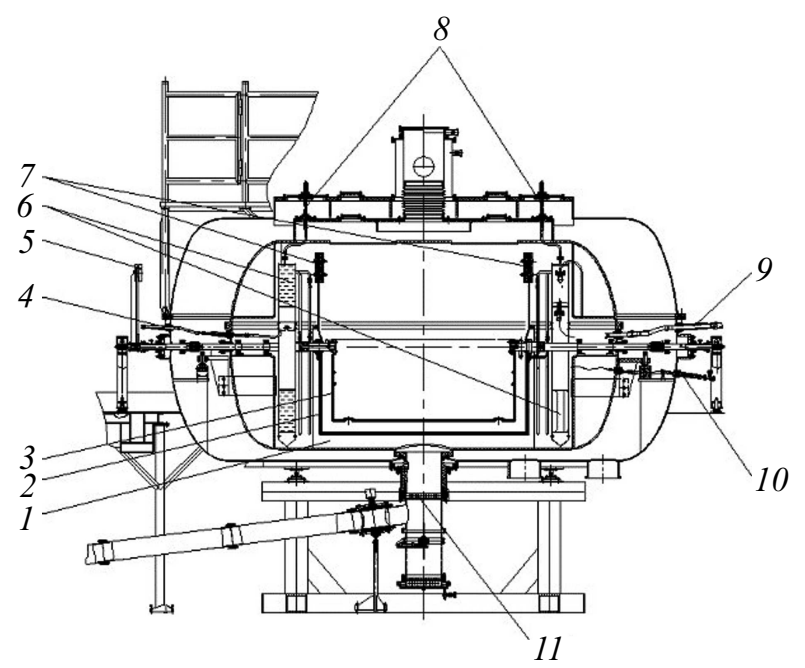

Рис. 3. Схема новой установки для измерения времени жизни нейтрона: 1 - внутренний объем накопления УХН, 2 ловушка для хранения УХН, 3 - вставка в ловушку для хранения УХН, 4 - выход гелия после охлаждения вставки, 5 - противовес вставки, 6 - емкости для жидкого гелия, 7 - противовесы ловушки, 8 - тракты заливки гелия в емкости, 9 - выход испаряющегося гелия из емкости, $10-$ выход гелия после охлаждения ловушки, $11-$ алюминиевая мембрана. 


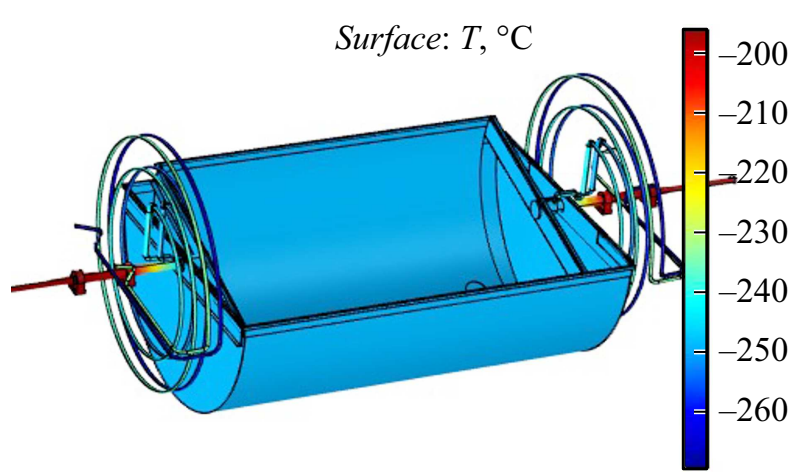

Рис. 4. Распределение температуры в материале ловушки и вставки.

величину $0.05 \mathrm{~W}$. Уровень теплопритока к емкостям с жидким гелием определяет скорость испарения и тем самым определяет поток газа, идущий на охлаждение ловушки или вставки. По нашим оценкам данный поток составит примерно $0.05 \mathrm{~g} / \mathrm{s}$, что соответствует примерно испарению 341 жидкого гелия в сутки.

Оценка теплопритока к ловушке и к вставке по валам оценивалась в предположении, что температура вала на расстоянии $50 \mathrm{~cm}$ от ловушки (вставки) составляет $77 \mathrm{~K}$. Полученная величина теплопритока $W_{\text {roll }} \approx 0.7 \mathrm{~W}$ может быть уменьшена за счет теплового контакта вала с медной трубкой, по которой еще холодный гелий после охлаждения ловушки (вставки) возвращается в линию сбора гелия. Оценка теплопритока к стенкам сосудов для хранения нейтронов за счет излучения дает примерно такую же величину. Учитывая высокую теплоемкость газообразного гелия $C=7.5 \mathrm{~J} /(\mathrm{g} \cdot \mathrm{K})$, при теплопритоке к ловушке (вставке) на уровне $1.5 \mathrm{~W}$ и потоке газа $0.05 \mathrm{~g} / \mathrm{s}$, температура стенок сосудов хранения будет превышать температуру испаряющегося газа на несколько градусов при условии достаточного теплообмена между газом, стенками трубок и материалом ловушки (вставки). Теплопроводность меди, из которой изготовлены сосуды хранения, достаточна для равномерного распределения температуры по поверхности. Результаты моделирования температурного распределения в материале ловушки и вставки представлены на рис. 4.

Для контроля температуры сосудов хранения используются кремниевые диоды, закрепленные на наружной поверхности ловушки в месте, удаленном от трубок охлаждения. При необходимости поток испаряющегося гелия может быть увеличен с помощью нагревателей, помещенных внутри емкостей с жидким гелием.

Изменения сечения охлаждающей трубки как сужение, так и расширение наряду с изгибами трубок приводят к увеличению местного сопротивления течению газа. Это означает, что для пропускания газа, испаряющегося из емкостей по трубкам охлаждения, закрепленным на ловушке и вставке, давление внутри емкостей должно превышать давление в линии сбора гелия примерно на 500 mbar. В этом случае конструкция, находящаяся в вакууме, должна выдерживать давление $0.15 \mathrm{MPa}$. Емкость для жидкого гелия имеет форму тороида с плоскими стенками. Диаметр внешней обечайки $1350 \mathrm{~mm}$, диаметр внутренней обечайки $675 \mathrm{~mm}$, ширина $135 \mathrm{~mm}$. Емкость изготовлена из полированной нержавеющей стали толщиной $1.5 \mathrm{~mm}$. Для обоснования устойчивости и механической прочности конструкции были проведены расчеты напряжений в материале. Схематическое изображение емкости для жидкого гелия и результаты расчета напряжений показаны на рис. 5.

Для увеличения механической прочности внутри емкости к боковым стенкам корпуса приварены две концентрические полосы с отверстиями для перетекания жидкого гелия и размещения уровнемера и технологических трубок, которые используются для заливки жидкого гелия и вывода испаряющегося газа. Внутри емкости предусмотрен нагреватель. Были проведены испытания емкости для жидкого гелия до избыточного давления $0.2 \mathrm{MPa}$, которые подтвердили надежность выбранной конструкции. Вес одной емкости для жидкого гелия равен примерно $35 \mathrm{~kg}$ и для первоначального охлаждения емкости от 77 до $4 \mathrm{~K}$ требуется снять тепло примерно 0.19 МЈ. Для этого необходимо испарение примерно 751 жидкого гелия. Этот испаряющийся газ будет использоваться для начального охлаждения ловушки и свинцовых противовесов, необходимых для балансировки ловушки, поворачивающейся с помощью вала, соединенного с шаговым двигателем. По оценкам теплоемкости, для начального охлаждения ловушки и противовесов необходимо снять тепло 2 МЈ. Для начального охлаждения вставки необходимо меньшее количество газа, так как противовес вставки расположен снаружи вакуумных кожухов и не требует охлаждения. Таким образом, для начального охлаждения двух емкостей необходимо примерно 1501 жидкого гелия, а для поддержания низ-

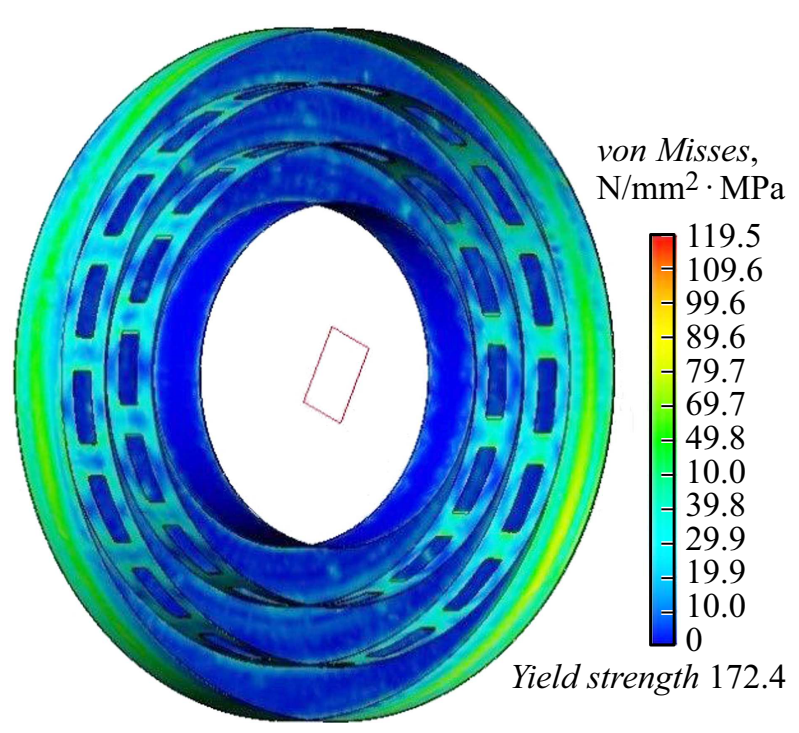

Рис. 5. Вид емкости для жидкого гелия с расчетами напряжений. 
кой температуры ловушки и вставки в ходе измерений потребуется примерно 351 жидкого гелия в сутки.

Измерение времени жизни нейтрона при удержании в ловушке, охлажденной до низкой температуры (примерно $10-15 \mathrm{~K}$ ), позволит улучшить точность определения этой фундаментальной величины в сравнении с измерениями, проведенными другими методами.

\section{Заключение}

В настоящий момент необходимые части экспериментальной установки изготовлены и планируется их монтаж. Авторы выражают благодарность О.П. Федоровой и Е.Н. Леоновой за конструкторскую разработку узлов установки. Авторы благодарны А.А. Костыгову и сотрудникам ЦЭТО ПИЯФ за помощь в изготовлении установки.

Работа выполнена при поддержке Российского Научного Фонда (Проект № 14-22-00105).

\section{Список литературы}

[1] Iocco F., Mangano G., Miele G. // et al. Phys. Repor. 2009. Vol. 472. N 1. P. $1-76$.

[2] Coc A. // Nucl. Instrum. Meth. A. 2009. Vol. 611. P. 224-230.

[3] Byrne J. // Rep. Progr. Phys. 1982. Vol. 45. P. 115-184.

[4] Schreckenbach K., Mampe W. // J. Phys. G: Nucl. Part. Phys. 1992. Vol. 18. P. 1-34.

[5] Dubbers D., Schmidt M.D. // Rev. Mod. Phys. 2011. Vol. 83. N 4. P. $1111-1171$.

[6] Mention G., Fechner M., Lasserre Th. // et al. Phys. Rev. D. 2011. Vol. 83. P. 073006.

[7] Zhang C., Qian X., Vogel P. // Phys. Rev. D. 2013. Vol. 87. P. 073018.

[8] Cirigliano V., Gardner S., Holstein B. // Prog. Part. Nucl. Phys. 2013. Vol. 71. P. 93-118.

[9] Byrne J., Dawber P. Spain J.A. // et al. Phys. Rev. Lett. 1990. Vol. 65. N 3. P. 289-292.

[10] Byrne J., Dawber P., Habeck C.G. // et al. Europhys. Lett. 1996. Vol. 33. N 3. P. 187-192.

[11] Nico J.S., Dewey M.S., Gilliam D.M. // et al. Phys. Rev. C. 2005. Vol. 71. P. 055502.

[12] Yue A.T., Dewey M.S., Gilliam D.M. // et al. Phys. Rev. Lett. 2013. Vol. 111. P. 222501.

[13] Serebrov A., Varlamov V., Kharitonov A. // et al. Phys. Lett. B. Vol. 605. P. 72-78.

[14] Серебров А.П., Коломенский Э.А., Фомин А.К. // и др. Письма в ЖЭТФ. 2017. Т. 106. Вып. 10.

[15] Mampe W., Ageron P., Dates C. // et al. Phys. Rev. Lett. 1989. Vol. 66. P. 593-596.

[16] Pichlmaier A., Varlamov V., Schreckenbach K. // et al. Phys. Rev. B. 2010. Vol. 693. P. 221-226.

[17] Arzumanov S., Bondarenko L., Chernyavsky S. // et al. NIM A. 2000. Vol. 440. P. 511-516.

[18] Arzumanov S., Bondarenko L., Chernyavsky S. // et al. Phys. Lett. B. 2015. Vol. 745. P. 79-89.

[19] Vladimirski V.V. // Sov. Phys. JETP. 1961. Vol. 12. P. 740.

[20] Paul W., Anton F., Paul L. // et al. Zeitsch. Phys. C. 1989. Vol. 45. P. 25-30.
[21] Ezhov V.F., Andreev A.Z., Ban G. // et al. NIM A. 2009. Vol. 611. P. 167-170.

[22] Salvat D.J., Adamek E.R., Barlow D. // Phys. Rev. C. 2014. Vol. 89. P. 052501(R).

[23] Серебров А.П., Фомин А.К., Харитонов А.Г. // и др. Кристаллография. 2016. Т. 61. Вып. 1. С. 152-156.

[24] Серебров А.П., Фомин А.К., Харитонов А.Г. // и др. ЖТФ. 2013. Т. 83. Вып. 11. С. 136-141. 